\title{
La eficacia clínica a largo plazo de los implantes con conexión interna y superficie arenada y grabada
}

\section{Long-term clinical efficacy of implants with internal connection and sandblasted-acid-etched surface.}

\author{
J. Gil González*, E. Núñez Márquez*, J. Moreno Muñoz*, N. Matos Garrido*,
} A. Jiménez Guerra**, L. Monsalve Guil ${ }^{* *}$, I. Ortiz García**, E. Velasco Ortega***

\section{RESUMEN}

Introducción. La implantología oral representa una opción importante en el tratamiento de los pacientes con pérdida dental total y parcial. El estudio a largo plazo muestra la evaluación de los pacientes tratados mediante carga precoz de implantes con conexión interna y superficie arenada y grabada.

Métodos. 40 pacientes con pérdidas dentales fueron tratados con implantes Frontier GMI ® con conexión interna y superficie arenada y grabada. Los implantes fueron cargados tras un periodo de tiempo de 6 semanas en la mandíbula y 8 semanas en el maxilar superior. Los hallazgos clínicos (implantológicos y prostodóncicos) se han seguido durante al menos 6 años.

Resultados. 103 implantes fueron insertados en ambos maxilares, 46 implantes (44,7\%) en el maxilar superior y 57 implantes $(55,3 \%)$ en la mandíbula, para su rehabilitación prostodóncica. 37 implantes $(35,9 \%)$ fueron insertados en el sector anterior y 66 implantes $(64,1 \%)$ en el sector posterior. 70 implantes (68\%) de forma sumergida (2 cirugías) y 33 implantes (32\%) de forma no sumergida (una cirugía). Después de un seguimiento clínico de 92,2 meses, los resultados indican una supervivencia de los implantes del 96,2\%; ya que se perdieron 4 implantes. La pérdida ósea marginal fué de 0,99 \pm ,84 mm. Las restauraciones prostodóncicas incluyeron 31 coronas unitarias, 15 puentes fijos, 5 sobredentaduras con bolas, 2 rehabilitaciones totales fijas y una rehabilitación completa híbrida. Las complicaciones tardías fueron 11 implantes $(10,7 \%)$ con periimplantitis y 6 prótesis $(11,1 \%)$ con complicaciones protésicas.

Conclusiones. Los hallazgos clínicos del estudio indican que el tratamiento con carga precoz mediante prótesis de los implantes con conexión interna y superficie arenada y grabada, representa una terapéutica odontológica con éxito.

PALABRAS CLAVE: Implantes dentales, superficie arenada y grabada, conexión interna, carga precoz, oseointegración, implantología oral.

\section{ABSTRACT}

Introduction. Implant dentistry constitute an important option in the prosthodontic treatment of patients

* $\quad$ Profesor Asistente Honorario de Odontología Integrada de Adultos y Gerodontologia. Profesor del Máster de Implantología Oral. Facultad de Odontología. Universidad de Sevilla.

* * Profesor Asociado de Odontología Integrada de Adultos y Gerodontologia. Profesor del Máster de Implantología Oral. Facultad de Odontología. Universidad de Sevilla.

*** Profesor Titular de Odontología Integrada de Adultos y Gerodontologia. Director del Máster de Implantología Oral. Facultad de Odontología. Universidad de Sevilla. 
with partial and total tooth loss. This long-term study reports the evaluation of patients treated by early loading of implants with internal connection and sandblasted-acidetched surface.

Methods. 40 patients with tooth loss were treated with Frontier GMI ${ }^{\circledR}$ sandblasted and acid-etched surface implants. Implants were loaded after a healing free-loading period of 6 weeks (mandible) and 8 weeks (maxilla). Clinical findings (implants and prosthodontics) were followed during at least 6 years.

Results. 103 implants were inserted (46 maxillary (44.7\%), and 57 mandibular (55.3\%)) for prosthodontic rehabilitation. 37 implants (35.9\%) were inserted in anterior sites and 66 implant (64.1\%) in posterior sites. Seventy implants (68\%) were placed submerged (two stages) while that 33 implants (32\%) were placed nonsubmerged (one stage). After at a mean follow-up of 92.2 months, clinical results indicate a survival rate of implants of $96.2 \%$. Four implants were lost during the treatment. Mean marginal bone loss were $0.99 \pm 0.84 \mathrm{~mm}$. Prosthodontic restorations included 31 single crowns, 15 fixed bridges, 5 overdentures, 2 fixed totally rehabilitation and an hybrid full rehabilitation. Delayed complications include 11 implants (10.7\%) with peri-implantitis and 6 prostheses (11.1\%) with technical complications.

Conclusions. Clinical results of this study indicate that prosthodontic rehabilitation by early loading of internal connection and sandblasted and etched- implants can be a successful dental treatment.

KEY WORDS: Dental implants, sandblasted and acid-etched surface, internal connection, early loading, osseointegration, implant dentistry.

Fecha de recepción: 10 de octubre de 2020

Fecha de aceptación: 12 de octubre de 2020

J. Gil González, E. Núñez Márquez, J. Moreno Muñoz, N. Matos Garrido, A. Jiménez Guerra, L. Monsalve Guil, I. Ortiz García, E. Velasco Ortega. La eficacia clínica a largo plazo de los implantes con conexión interna y superficie arenada y grabada. 2021; 37, (1): 11-18.

\section{INTRODUCCIÓN}

Los implantes dentales han representado en los últimos 35 años, el tratamiento más importante para la rehabilitación de los dientes perdidos ${ }^{1}$. En el transcurso de las últimas décadas, se ha establecido los fenómenos biológicos que configuran la oseointegración y que desarrollan una conexión funcional y estructural directa entre el hueso vivo y la superficie del implante sometido a cargas. La investigación experimental indica la alta predictibilidad de la interfase hueso-implante mediante estudios histológicos que demuestran la importancia de la superficie de los implantes para conseguir la oseointegración ${ }^{2-3}$. La superficie arenada y grabada es la más utilizada en los sistemas implantes actuales, presentando una gran evidencia científica fundamentada en estudios en animales y clínicos con pacientes ${ }^{4-7}$.

La cirugía de implantes ha representado un aspecto esencial del tratamiento integral del paciente con pérdidas dentales de su dentición natural. Mediante la cirugía de implantes, el profesional coloca las raíces artificiales que posteriormente van a soportar las prótesis correspondientes ${ }^{8-9}$. La localización, el número, y el tamaño (diámetro y longitud) de los implantes está determinada por la evaluación preoperatoria del paciente, por una posición óptima funcional de acuerdo al tipo de prótesis dental seleccionada y a los aspectos anatómicos del proceso alveolar en relación al volumen y densidad del hueso disponible ${ }^{9 .}$

Las conexiones externas e internas han supuesto la mayoría de los diseños establecidos para la unión de los implantes con sus correspondientes prótesis ${ }^{10}$. La conexión interna surgió para solventar todos los problemas derivados del uso de la conexión externa, buscando una mayor estabilidad a nivel de la unión implante-pilar, un mejor sellado biológico antibacteriano y un menor microgap ${ }^{11-12}$

Desde los comienzos de la implantología oral, la evolución de la prostodoncia sobre implantes ha sido innovadora y progresiva. La unión de los pilares conectados a los implantes influye en la función y la estética del paciente, además la unión pilar-implante es fundamental en el mantenimiento de los tejidos periimplantarios ${ }^{13-14}$.

Clásicamente, la experiencia clínica había demostrado que un periodo mínimo de 3-6 meses de cicatrización ósea con los implantes era imprescindible para 


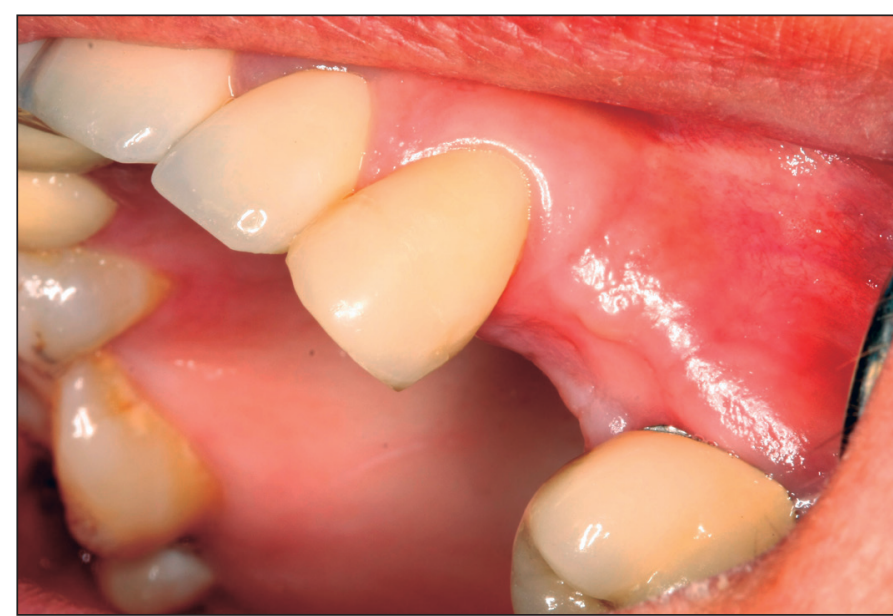

Figura 1. Aspecto clínico del reborde alveolar superior izquierdo previo a la cirugía de implante.

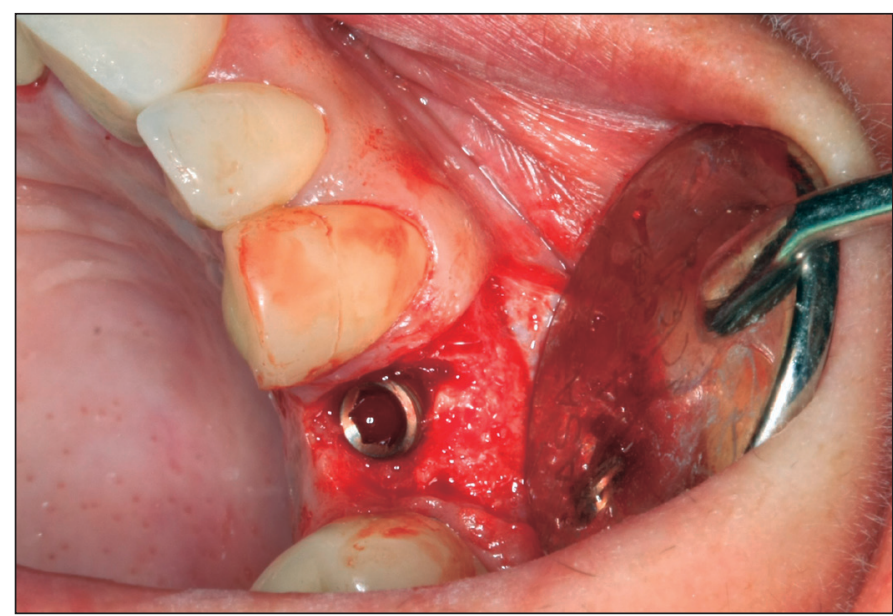

Figura 3. Aspecto clínico del implante de conexión interna insertado.

conseguir la oseointegración y posteriormente la carga funcional con la prótesis correspondiente ${ }^{15-16}$. Sin embargo, la investigación experimental y clínica ha desarrollado diferentes tipos de carga funcional como la carga precoz. En este sentido, la carga precoz puede ser realizada mediante una selección cuidadosa de cada caso con buena calidad y cantidad de hueso y unas técnicas quirúrgica y prostodóncica adecuadas que asegure una respuesta favorable a corto y largo plazo de los pacientes tratados ${ }^{17-18}$.

Los estudios a largo plazo indican que la fase de mantenimiento debe incluir las revisiones periódicas del tratamiento con implantes y debe basarse en una colaboración permanente entre el profesional y el paciente ${ }^{19}$. El profesional debe conocer la longevidad de las intervenciones quirúrgicas y prostodóncicas realizadas, y previamente al comienzo del tratamiento informar al paciente e incluir esta información en el consentimiento informado ${ }^{20-21}$.

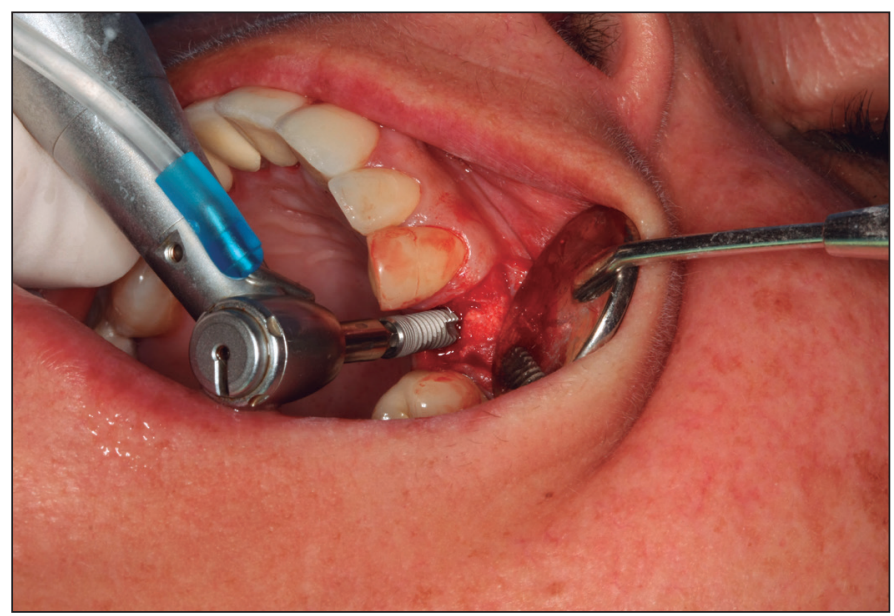

Figura 2. Cirugía con colgajo para inserción de implante en sustitución del primer premolar superior izquierdo.

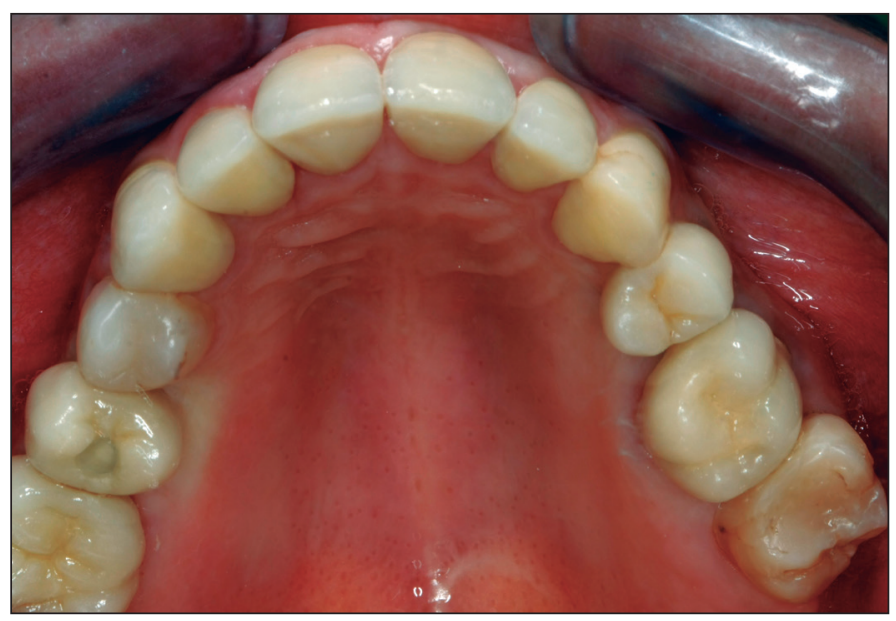

Figura 4. Aspecto clínico de la corona cementada sobre el implante.

El objetivo del presente estudio era la evaluación y el seguimiento clínico del tratamiento con implantes de conexión interna con superficie arenada y grabada, cargados precozmente con diferentes tipos de prótesis.

\section{PACIENTES Y METODOS.}

El presente estudio fué realizado por profesores de las unidades docentes de Odontología Integrada de Adultos y del Máster de Implantología Oral de la Facultad de Odontología de la Universidad de Sevilla.

Previamente a la realización del estudio, aquellos pacientes que padecían trastornos sistémicos graves que pudieran afectar a la oseointegración fueron excluidos del estudio. Los pacientes seleccionados eran adultos, de ambos sexos. Todos los pacientes presentaban pérdidas dentales unitarias, parciales o totales y fueron informados del protocolo del tratamiento con implantes, 
de los aspectos quirúrgicos y prostodóncicos, temporalización del tratamiento y seguimiento, así como de la posibilidad de la existencia de complicaciones y pérdida de implantes, autorizando el tratamiento implantológico mediante un consentimiento informado. Antes del tratamiento, todos los pacientes fueron evaluados radiológicamente, con una ortopantomografía y en los casos necesarios con una tomografía computarizada de haz cónico.

Los criterios de éxito y supervivencia de los implantes fueron los recomendados por van Steenberghe y cols. ${ }^{22}$. En este sentido, la supervivencia fué definida como la presencia permanente de los implantes en su localización original aunque no tengan valor clínico o cause efectos adversos.

CIRUGIA. Una hora antes de la cirugía, los pacientes comenzaron un régimen antibiótico preventivo (amoxicilina + clavulánico) durante una semana. Todos los pacientes recibieron anestesia local. Todos los implantes fueron insertados de forma diferida, tras un periodo mínimo de 6 meses después de la extracción dental. Todos los implantes utilizados en el presente estudio eran Frontier $®$ (Global Medical Implants, Barcelona, España) roscados de conexión interna y de superficie arenada y grabada. Todos los implantes fueron estables después de la inserción. La preparación del lecho y la inserción de los implantes se realizó según el protocolo estandarizado convencional con fresas consecutivas de menor a mayor diámetro, a una velocidad constante de 800 r.p.m. (Figuras 1-3) A la semana se retiraron las suturas y a los pacientes se les recomendó el enjuague diario con clorhexidina durante los primeros 30 días. En los casos de implantes sumergidos, se realizó la segunda cirugía, previamente a la carga de los implantes, con la colocación del correspondiente tornillo o pilar de cicatrización.

PROSTODONCIA. A las 6 semanas (en la mandíbula) y a las 8 semanas (en el maxilar superior) después de la inserción de los implantes (Figura 4), se realizó la carga precoz funcional de los implantes mediante la colocación de los pilares protéscios sobre las plataformas reducidas, y posteriormente, las correspondientes prótesis implantosoportadas. El seguimiento clínico de los pacientes tratados desde la carga funcional de los implantes fué al menos de 6 años.

ANALISIS ESTADISTICO. Se realizó una estadística descriptiva de los hallazgos clínicos del estu-

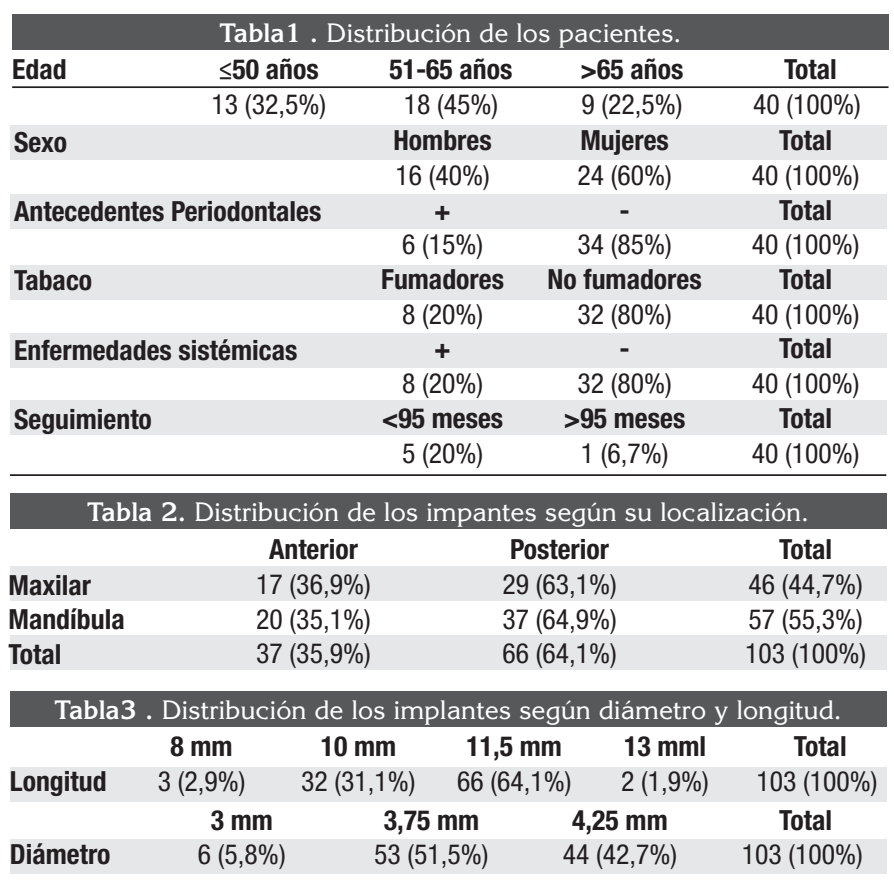

dio, las variables demográficas y clínicas de los pacientes, la supervivencia, las complicaciones y pérdidas de los implantes así como las restauraciones prostodóncicas realizadas. Las variables cualitativas fueron analizadas con el test de la chi-cuadrado, y las variables cuantitativas según el test de la varianza. Cuando la distribución de las variables cualitativas no fue normal, se utilizaron el test de la $U$ de Mann-Whitney para las variables dicotómicas y el test de Kruskal-Wallis para las variables con más de dos categorías.

\section{RESULTADOS}

PACIENTES. 40 pacientes con pérdidas dentales (24 mujeres y 16 hombres) participaron en el estudio, con una edad media de 54,4 años (rango: 24-71 años). 8 pacientes $(20 \% \%)$ eran edéntulos totales. 6 pacientes $(15 \%)$ presentaban antecedentes periodontales. 8 pacientes (20\%) eran fumadores y 8 pacientes (20\%) presentaban alguna enfermedad sistémica (hipertensión arterial, cardiopatía) aunque controlada (Tabla 1).

IMPLANTES. Se insertaron un total de 103 implantes Frontier GMI ® en los correspondientes 40 pacientes. 46 implantes $(44,7 \%)$ fueron insertados en el maxilar superior y 57 implantes $(55,3 \%)$ en la mandíbula, para su rehabilitación prostodóncica. 37 implantes (35,9\%) fueron 
insertados en el sector anterior (sustitución de incisivos y caninos) y 66 implantes $(64,1 \%)$ en el sector posterior (sustitución de premolares y molares) (Tabla 2).

De los implantes utilizados, 6 fueron de 3,3 $\mathrm{mm}$ de diámetro; 53 de $3,75 \mathrm{~mm}$ de diámetro y 44 fueron de $4,25 \mathrm{~mm}$ de diámetro. Con respecto a la longitud, fueron insertados 66 implantes de 11,5 mm, 32 de 10 $\mathrm{mm}, 3$ de $8 \mathrm{~mm}$ y 2 de $13 \mathrm{~mm}$ (Tabla 3 ).

PROTESIS IMPLANTOSOPORTADAS. Se realizaron 54 prótesis en los 40 pacientes. 31 coronas unitarias (57,4\%), 15 prótesis parciales fijas (27,8\%), 5 sobredentaduras retenidas por bolas $(9,3 \%), 2$ rehabilitaciones totales fijas $(3,7 \%)$ y una rehabilitación total híbrida $(1,8 \%)$ (Tabla 4). De las 49 prótesis fijas, 25 (51\%) fueron cementadas y el resto, 24 (49\%) fueron atornilladas.

\section{SEGUIMIENTO CLINICO.}

Después de un periodo medio de seguimiento clínico de 92,2 meses, 4 implantes se perdieron (3,8\%). Un implante se perdió de forma temprana durante la fase de cicatrización. Posteriormente, 3 implantes se perdieron después de la carga funcional durante el seguimiento clínico. La pérdida ósea media marginal fué de $0,99 \pm 0,84 \mathrm{~mm}$.

Como complicaciones tardías hubo 11 implantes con periimplantitis $(10,6 \%)$ que fueron tratados con regeneración ósea. 6 prótesis presentaron complicaciones prostodóncicas $(11,1 \%)$.

\section{DISCUSION}

El objetivo fundamental del presente estudio clínico prospectivo a más de 7 años, era obtener y mantener una nivel favorable de oseointegración de los implantes con conexión interna y superficie arenada y grabada, una estabilidad de los tejidos blandos periimplantarios para conseguir unos buenos resultados funcionales y estéticos mediante la carga precoz con sus correspondientes prótesis. En este sentido, los hallazgos clínicos indican una tasa de éxito del 96,2\%, lo que confirma la eficacia de la carga precoz con las diversas prótesis correspondientes de los implantes dentales utilizados con un diseño macroscópico de conexión interna y una superficie arenada y grabada, insertados mediante una o dos cirugías, en los pacientes con pérdidas dentales unitarias, parciales y totales.
El éxito elevado del presente estudio en primer lugar está relacionado con la selección de los pacientes, su diagnóstico y la planificación de tratamiento. Todos los pacientes seleccionados fueron adultos que desde un punto de vista médico, no padecían enfermedades sistémicas graves que pudieran comprometer la oseointegración y el resultado del tratamiento implantológico. Solamente el $20 \%$ de los pacientes presentaban alguna enfermedad sistémica o eran fumadores. El consumo de tabaco constituye un factor importante de riesgo para el tratamiento con implantes, ya que reduce la tasa de oseointegración y está relacionado directamente con un mayor número de fracasos ${ }^{23-24}$.

Entre los 103 implantes insertados, el 68\% fué insertado de forma sumergida (2 fases) y el $32 \%$ de forma no sumergida (una fase), demostrando que ambas técnicas quirúrgicas después de un periodo de seguimiento clínico de más de 7 años, presentaron una elevada tasa de éxito (97,2\% vs 93,9\%). La evidencia científica demuestra que los resultados funcionales y estéticos con los diferentes sistemas de implantes utilizando ambas técnicas quirúrgicas, sumergida son muy aceptables 25-29. No obstante, la técnica no sumergida o de una cirugía ha ido ganando popularidad y prestigio entre los implantólogos porque reduce las intervenciones quirúrgicas al paciente, y los tejidos periimplantarios cicatrizan muy favorablemente para la posterior rehabilitación prostodóncica ${ }^{29}$.

Del total de los implantes insertados, 57 implantes fueron insertados en la mandíbula y 46 implantes en el maxilar superior. Algunos estudios han expresado que las tasas de supervivencia y éxito de los implantes son menores en el maxilar superior que en la mandíbula 30-31. Incluso se ha demostrado en revisiones sistemáticas, el doble de fracasos en los implantes insertados en el maxilar superior comparados con la mandíbula ${ }^{31}$. En el presente estudio, el 4,3\% de los implantes maxilares y el 3,5\% de los mandibulares, fracasaron . En el presente estudio, la mayoría de los pacientes presentaban pérdidas unitarias o parciales y la presencia de dientes naturales adyacentes a los implantes pueden ayudar a retrasar la reabsorción alveolar, y favorecer una protección oclusal comparados con los implantes insertados en tramos edéntulos totales ${ }^{32-33}$.

El 64,1\% de los implantes fué insertado en el sector posterior y el 35,9\% en el sector anterior. El sector posterior representa el área ideal para valorar el éxito biomecánico de los implantes porque son sometidos a mayores fuerzas masticatorias y las cargas oclusales y parafuncionales se concentran más en esta región ${ }^{33-34}$. 
En este sentido, los implantes de diámetro más ancho pueden conseguir una mayor interfase titanio-hueso y son más resistentes que los implantes estándares ${ }^{35}$.

En el sector anterior, el compromiso estético es de gran importancia para los tejidos duros y blandos periimplantarios, ya que los implantes suelen sustituir dientes anteriores que se han perdido por traumatismos, fracasos endodóncicos o enfermedad periodontal avanzada. En estos casos, los implantes representan la mejor terapéutica actual porque las coronas unitarias implantosoportadas ofrecen una buena función y estética, y la respuesta del paciente al tratamiento es muy positiva ${ }^{18,36}$.

La estructura y el diseño macroscópico de los implantes puede estar también relacionado con el éxito del tratamiento ${ }^{37-38}$. En el presente estudio, los implantes presentaban longitudes entre 8 y $13 \mathrm{~mm}$ y el diámetro variaba entre los $3,3 \mathrm{~mm}$ y los $4,25 \mathrm{~mm}$, aunque la mayoría de los implantes tenían características macroscópicas estándar. De hecho, la longitud de los implantes puede influir en el éxito de los tratamientos, es decir, a mayor longitud del implante dental, existirá una mayor superficie de contacto, lo que permitirá aumentar el ratio de contacto entre hueso e implante, por ejemplo, un implante de $10 \mathrm{~mm}$ aumenta un $30 \%$ su área de contacto respecto a un implante de $7 \mathrm{~mm}^{37}$. También influye en la oseointegración el diámetro del implante dental. Un mayor diámetro implantario asegura una mayor superficie de contacto con el hueso, lo que asegurará una mejor respuesta biomecánica. Los estudios señalan que el aumento de diámetro influye más positivamente en la integración del implante que el aumento de la longitud del mismo, aunque no es capaz de compensar la falta de longitud de los implantes $\operatorname{cortos}^{38}$.

El macrodiseño de los implantes y su influencia en los resultados clínicos del tratamiento debe ser interpretado por el implantólogo en su integridad. La estabilidad primaria óptima y la concentración de estrés biomecánico son resultado de factores biológicos y mecánicos 39. La incorporación de espiras de menor tamaño en el área cervical, como presentan los implantes insertados en el presente estudio de investigación doctoral, ha demostrado un incremento de su capacidad para resistir fuerzas axiales y un estímulo biomecánico para ayudar a preservar el hueso marginal periimplantario ${ }^{40}$.

Los implantes de conexión interna pueden obtener una excelente fijación y transmisión de fuerzas de los pilares a los implantes. Estos hallazgos clínicos son confirmados por el presente estudio, donde los im- plantes utilizados presentaban una conexión interna para su unión a los correspondientes pilares protésicos 18-19. Un aspecto importante, también del diseño de los implantes utilizados en este estudio es que presentaban en su diseño una plataforma reducida o platform switching. Este concepto se refiere a la utilización de pilares con un diámetro más reducido que el de la plataforma del implante, es decir, se incrementa la distancia horizontal entre la unión pilar-implante y la interfase hueso-implante ${ }^{41-42}$. El concepto de plataforma reducida o platform switching fué desarrollado para controlar la pérdida de hueso periimplantario después de la inserción de los implantes y ha sido demostrado en estudios experimentales con animales y en estudios clínicos con pacientes con buenos resultados a largo plazo. La plataforma reducida disminuye las fuerzas oclusales y la contaminación bacteriana en la interfase entre el hueso crestal y el implante ${ }^{41-42}$.

El presente estudio valora los resultados clínicos de la utilización de implantes con una superficie arenada y grabada que han demostrado una rugosidad óptima que estimula la oseointegración y consiguen una mayor respuesta celular con adhesión, proliferación y diferenciación de los osteoblastos ${ }^{6-7}$. La superficie arenada y grabada incrementa el área de contacto durante la fase de cicatrización con una favorable respuesta ósea que mejora la fijación del implante desde un punto de vista biomecánico ${ }^{6-7}$.

En este sentido, los resultados del presente estudio demuestra la alta predictibilidad de la superficie arenada y grabada en conseguir su oseointegración. De los 103 implantes insertados, solamente un implante $(0,9 \%)$ fracasó de forma inmediata y posteriormente, durante el periodo de 7,7 años de seguimiento clínico fracasaron 3 implantes más (2,9\%). La tasa global definitiva de éxito del tratamiento fué del $96,2 \%$.

Los protocolos de carga precoz se han desarrollado, fundamentalmente, porque ha mejorado el conocimiento de la oseointegración que ha ayudado a desarrollar nuevos diseños de los implantes modernos. Las espiras cervicales y la superficie arenada y/o grabada) han logrado incrementar un buen contacto hueso-implante y una rápida oseointegración 9,17-19. También se han desarrollado nuevos materiales y aditamentos protésicos así como mayores niveles de precisión y ajuste que han logrado una mejor unión pilar-implante, y han reducido también el tiempo de espera de la carga funcional convencional a protocolos de carga precoz con unos resultados funcionales y estéticos muy favorables ${ }^{43-44}$. En el presente estudio, se realizó la 
J. Gil González, E. Núñez Márquez, J. Moreno Muñoz, N. Matos Garrido,

A. Jiménez Guerra, L. Monsalve Guil, I. Ortiz García, E. Velasco Ortega La eficacia clínica a largo plazo de los implantes con conexión interna y superficie arenada y grabada.

carga precoz, a las 6 semanas en la mandíbula y a las 8 semanas en el maxilar superior, en todos los tipos de restauraciones sobre implantes, coronas unitarias $(57,4 \%)$, puentes fijos $(27,8 \%)$, rehabilitaciones totales $(5,5 \%)$ y sobredentaduras $(9,3 \%)$.

La pérdida de hueso marginal representa un parámetro biológico y clínico importante para definir el éxito a largo plazo de los implantes ${ }^{45}$. En este sentido, en el presente estudio a 7,7 años, la pérdida de hueso crestal media fue de 0,99 0,84 mm. En el presente estudio se observó periimplantitis en 11 implantes (10,6\%) con mayor incidencia en pacientes con antecedentes periodontales y fumadores, lo que confirman a estas condiciones como importantes factores de riesgo de la periimplantitis ${ }^{46-47}$.

Los resultados clínicos del presente estudio demostraron una prevalencia de complicaciones en 6 prótesis $(11,1 \%)$ durante el periodo de 7,7 años transcurridos desde la carga, que no afectaron a la supervivencia de las prótesis. Las prótesis cementadas suelen presentar más complicaciones biológicas inflamatorias por el exceso de cemento y las atornilladas más técnicas, como aflojamiento y fracturas de los tornillos de conexión, aunque presentan la ventaja de que pueden retirarse fácilmente ${ }^{48-49}$.

\section{CONCLUSIONES}

El tratamiento con implantes se ha convertido en una opción restauradora predecible a largo plazo. Un aspecto fundamental del éxito es conseguir y mantener un nivel adecuado de oseointegración de los implantes con una superficie rugosa. La cirugía con implantes con una macrogeometria favorable para lograr un grado importante de estabilidad primaria y un protocolo de carga precoz para la rehabilitación prostodóncica pueden representar el éxito del tratamiento.

\section{REFERENCIAS BIBLIOGRÁFICAS}

1. Balshi TJ, Wolfinger GJ, Balshi SF, Bidra AS. A 30-year follow-up of a patient with mandibular complete-arch fixed implant-supported prosthesis on 4 implants: a clinical report. J Prosthod 2019; 28: 97-102.

2. Papaspyridakos $P$, Chen CJ, Singh $M$, Weber HP, Gallucci GO. Success criteria in implant dentistry: a systematic review. $J$ Dent Res 2012; 91:242-248.

3. Gehrke S, Bragança LK, Velasco-Ortega E, Calvo-Guirado JL. Evaluation of dimensional behavior of peri-implant tissues in implants immediately exposed or submerged in fresh extraction and healed sites: a histological study in dogs. Int J Impl
Dent 2018; 4:5.

4. Rupp F, Lianga L, Geis-Gerstorfer J, Scheideler L, Hüttig F. Surface characteristics of dental implants: A review. Dent Mater 2018; 34: 40-57.

5. Jemat A, Ghazali, MJ. Razali M, Otsuka Y. Surface modifications and their effects on titanium dental implants. BioMed Res Int 2015, 791725.

6. Ramaglia L, Postiglione L, di Spigna G, Capece G, Salzano S, Rossi G. Sandblasted-acid-etched titanium surface influences in vitro the biological behavior of SaOS-2 human osteoblast-like cells. Dent Mater J 2011; 30:183-192.

7. Velasco-Ortega E, Ortiz-García I, Jiménez-Guerra J, Monsalve-Guil L, Muñoz-Guzón F, Gil FJ. Comparison between sandblasted-acid etched and oxidized titanium dental implants: In vivo study. J Mol Sci 2019; 20:13.

8. Yeniyol S, Jimbo R, Marin C, Tovar N, Janal MN, Coelho PG. The effect of drilling speed on early bone healing to oral implants. Oral Surg Oral Med Oral Pathol Oral Radiol 2013;116:550-555.

9. Galarza Estebaranz P, Díaz Prada FJ, López Castro JJ, Pérez Fontal V. La rehabilitación fija sobre implantes en pacientes edéntulos totales. Un estudio a dos años. Av Perio Impl 2015; 27, 3: 135-144.

10. Finger IM, Castellon P, Block M, Elian N. The evolution of external and internal implant/abutment connections. Pract Proced Aesthet Dent. 2003;15:625-632.

11. Niznick GA. The implant abutment connection: The key to prosthetic success. Compend Cont Educ Dent 1991;12:932937.

12. Binon PP. The evolution and evaluation of two interference-fit implant interfaces. Postgraduate Dent 1996; 3:3-13.

13. Montero J, Fernández-Ruiz J, Pardal-Peláez B, Jiménez-Guerra A, Velasco-Ortega E, Nicolas-Silvente A, Monsalve-Guil L. Effect of rough surface platforms on the mucosal attachment and the marginal bone loss of implants: A dog study. Materials 2020;13: 802.

14. Haidar Wehbe A, Matos Garrido N, Ayllón Guerola JM, Moreno Muñoz J, Núñez Márquez E, Velasco-Ortega E. El efecto de la fatiga cíclica sobre los pilares de implantes dentales. Av Odontoestomatol 2020;36: 89-97.

15. Adell R, Eriksson B, Lekholm U, Branemark PI, Jemt T. A long-term follow-up study of osseointegrated implants in the treatment of totally edentulous jaws. Int J Oral MaxilloFac Implants 1990, 5: 347 - 359.

16. Wismeijer D, Van Waas MAJ, Vermeeren JIJF, Mulder J, Kalk W. Patient satisfaction with implant-supported mandibular overdentures. A comparison of three treatment strategies with ITI-dental implants. Int J Oral Maxillofac Surg 1997, 26: 263267.

17. Liu J, Huang Q, Wang X, Li Y, Zhou J, Zeng D, et al. Early loading of splinted implants in posterior mandible: Three-year results of a prospective multicenter study. Clin Oral Implants Res 2019; 30:1049-1058.

18. Velasco Ortega E, Monsalve Guil L, Matos Garrido N, Jiménez Guerra A, García Méndez A, Medel Soteras R, Ortiz García I, España López A. La carga precoz de los implantes GMI con conexión interna y superficie arenada y grabada. Av Perio Impl 2014; 26: 67-75.

19. Velasco-Ortega E, Jiménez-Guerra A, Monsalve-Guil L, Ortiz-Garcia I, Nicolas-Silvente AI, Segura-Egea JJ, López-Lopez J. Long-term clinical outcomes of treatment with dental 
implants with acid etched surface. Materials 2020; 13:1553.

20. Todescan S, Lavigne S, Kelekis-Cholakis A. Guidance for the maintenance care of dental implants: clinical review. J Can Dent Assoc 2012; 78:107.

21. Kashbour WA, Rousseau NK, John Mark Thomason JM, Ellis JS. Provision of information on dental implant treatment: Patients' thoughts and experiences. Clin Oral Impl Res 2018;29:309-319.

22. van Steenberghe D, Quirynen M, Naert I. Survival and success rates with oral endosseous implants. En: Lang NP, Karring T, Lindhe $\mathrm{J}$ (eds.). Proceedings of the 3rd European Workshop on Periodontology. Implant Dentistry. Berlin: Quintessence. 1999. pag: 242-252.

23. Mohanty R, Sudan PS, Dharamsi AM, Mokashi R, Misurya AL, Kaushal P. Risk assessment in long-term survival rates of dental implants: a prospective clinical study. J Contemp Dent Pract 2018 ;19: 587-590.

24. Ting M, Craig J, Balkin BE, Suzuki JB. Peri-implantitis: a comprehensive overview of systematic reviews. J Oral Implantol 2018; 44:225-247.

25. Branemark PI, Hansson BO, Adell R, et al. Osseointegrated implants in the treatment of edentulous jaw. Experience for 10 -years period. Scand J Plast Reconstr Surg 1977;11(Suppl 16).1-132.

26. Buser D, von Arx T, ten Bruggenkate $C$, Weingart D. Basic surgical principles with ITI implants. Clin Oral Impl Res 2000; 11 (suppl 1): 59-68.

27. Buser $D$, Weber HP, Bragger $U$, Balsiger $C$. Tissue integration of one-stage ITI implants: 3-year results of a longitudinal study with hollow-cylinder and hollow-screw implants. Int J Oral MaxilloFac Implants 1991, 6: 405 - 412.

28. Collaert B, De Bruyn H. Comparison of Branemark fixture integration and short-term survival using one stage or two-stage surgery in completely and partially edentulous mandibles. Clin Oral Impl Res 1998; 9:131-135.

29. Eliasson A, Narby B, Ekstrand, K, Hirsch J, Johansson A, Wennerberg A. A 5-year prospective clinical study of submerged and nonsubmerged Paragon system implants in the edentulous mandible. Int J Prosthodont 2010; 23:231-238.

30. Krisam J, Ott L, Schmitz S, Klotz AL, Seyidaliyeva A, Rammelsberg P, Zenthöfer A. Factors affecting the early failure of implants placed in a dental practice with a specialization in implantology - a retrospective study. BMC Oral Health 2019: 19:208.

31. Moy PK, Medina D, Shetty V, Aghaloo TL. Dental implant failure rates and associated risk factors. Int $\mathrm{J}$ Oral Maxillofac Implants 2005; 20:569-577.

32. Klineberg I, Trulsson M, Murray G. Occlusion on implants-is there a problem. J Rehabil 2012; 39:522-537.

33. Abichandani SJ, Bhojaraju N, Guttal S, Srilakshmi J. Implant protected occlusion: A comprehensive review. Eur J Prosth 2013; 1:29-36.

34. Liu J, Huang Q, Wang X, Li Y, Zhou J, Zeng D, et al. Early loading of splinted implants in posterior mandible: Three-year results of a prospective multicenter study. Clin Oral Implants Res 2019; 30:1049-1058.

35. Lee CT, Chen YW, Starr JR, Chuang SK. Survival analysis of wide dental implant: systematic review and meta-analysis. Clin Oral Impl Res 2016; 27:1251-1264.

36. Wojtovicz E, España López A, Jiménez Guerra A, Ortiz García
I, Matos Garrido N, Monsalve Guil L, Velasco Ortega E. Seguimiento clínico de cuatro años de implantes unitarios anteriores insertados en alveolos postextracción. Av Odontoestomatol 2017; 33: 239-246.

37. Guan H, van Staden R, Loo YC, Johnson N, Ivanovski S, Meredith $\mathrm{N}$. Influence of bone and dental implant parameters on stress distribution in the mandible: a finit element study. Int $\mathrm{J}$ Oral Maxillofac Implants 2009;24: 866-876.

38. Ortega-Oller I, Suárez F, Galindo-Moreno P,Torrecillas-Martínez L, Monje A, Catena A, Hom-Lay Wang H. The influence of implant diameter on its survival: a meta-analysis based on prospective clinical trials. J Periodontol 2014;85: 569-580.

39. Ryu HS, Namgung C, Lee JH, Lim YJ. The influence of thread geometry on implant osseointegration under immediate loading: a literature review. J Adv Prosth 2014; 6:547-554.

40. Kang YI, Lee DW, Park KH, Moon IS. Effect of thread size on the implant neck area: preliminary results at 1 year of function. Clin Oral Impl Res 2012;23: 1147-1151.

41. Bish González MJ, Ortiz-García I, Jimenez-Guerra A, Monsalve Guil L, Matos Garrido N, Velasco-Ortega E. La respuesta tisular a implantes dentales con plataforma reducida (platform switching). Av Odontoestomatol 2020;36: 107-115.

42. Canullo L, Fedele GR, Iannello G, Jepsen S. Platform switching and marginal bone-level alterations: the results of a randomized-controlled trial. Clin Oral Implants Res 2010; 21:115-121.

43. Gallardo YNR, da Silva-Olivio IR, Gonzaga L, Sesma N, Martin W. A systematic review of clinical outcomes on patients rehabilitated with complete-arch fixed implant-supported prostheses according to the time of loading. $J$ Prosthodont 2019;28:958-968.

44. Abduo J, Lyons K, Bennani V, Waddell N, Swain M. Fit of screw-retained fixed implant frameworks fabricated by different methods: a systematic review. Int J Prosthodont 2011;24:207-220.

45. Naveau A, Shinmyouzu K, Moore C, Avivi-Arber L, Jokerst $\mathrm{J}$, Koka S. Etiology and measurement of peri-implant crestal bone loss (CBL). J Clin Med 2019; 8:166.

46. Stacchi C, Berton F, Perinetti G, Frassetto A, Lombardi T, Khoury A, Andolsek F, Di Lenarda R. Risk factors for peri-implantitis: effect of history of periodontal disease and smoking habits. A systematic review and meta-analysis. J Oral Maxillofac Res 2016; 7:3.

47. Tsigarida AA, Dabdoub SM, Nagaraja HN, Kumar PS. The influence of smoking on the peri-implant microbiome. J Dent Res 2015; 94:1202-1217.

48. Lin CY, Chen Z, Pan WL, Want HL. The effect of supportive care in preventing peri-implant diseases and implant loss: A systematic review and meta-analysis. Clin Oral Implants Res 2019; 30:714-724.

49. Gaddale R, Mishra SK, Chowdhary R. Complications of screwand cement-retained implant-supported full-arch restorations: a systematic review and meta-analysis. Int $\mathrm{J}$ Oral Implantol 2020; 13:11-40.

\section{CORRESPONDENCIA:}

Dr. Eugenio Velasco Ortega

Facultad de Odontología

$\mathrm{C} /$ Avicena s/n

41009 Sevilla

e-mail: evelasco@us.es 